\title{
Myocardial Strain Analysis using CMR Feature Tracking - The Good, The Bad and The Ugly
}

\author{
David Mui' and Yuchi Han²
}

\begin{abstract}
${ }^{1}$ Perelman School of Medicine, University of Pennsylvania, Philadelphia, Pennsylvania, USA
${ }^{2}$ Cardiovascular Division, Department of Medicine, Perelman School of Medicine, University of Pennsylvania, Philadelphia, Pennsylvania, USA
\end{abstract}

Corresponding author:

Yuchi Han MD, MMSc, Cardiovascular Division, Perelman School of Medicine, 11-135 South Pavilion

3400 Civic Center Blvd, Philadelphia, PA 19104

Received 19 Januar 2020; accepted 21 Februar 2020

Myocardial strain analysis has emerged as a valuable diagnostic and prognostic tool for cardiac disease detection and treatment. Currently, the gold standard for the non-invasive measurement of strain is using cardiovascular magnetic resonance imaging (CMR). While there are multiple ways to assess strain, including myocardial tagging (MT) and displacement encoding with stimulated echoes (DENSE), CMR feature-tracking (FT) has emerged as a simple post-processing tool for quantifying strain in large data sets. Because FT does not require acquisition of specific sequences, it has the ability to be used in large quantities of already acquired steady-state free procession (SSFP) cine images. However, despite its advantages, CMR FT also has numerous disadvantages. Acquisition-based techniques like MT and DENSE have better reproducibility in strain measurements than FT. FT is also limited by its spatial and temporal resolution as pixel size may affect the tracking of myocardial deformations. Furthermore, FT is limited by inter-vendor differences in the methods of calculating strain. Different vendors produce different strain values and there is currently no standardization of cine parameters or understanding of the performance of the different software with specific reference values. Future standardization of FT post-processing software must be implemented to interpret the results of FT in clinical and research settings.

Keywords: Feature tracking, strain, CMR

\section{Introduction}

Accurate assessment of cardiac function is the cornerstone of cardiac disease detection and treatment. Left ventricular ejection fraction (LVEF), often used as a marker for overall cardiac function, is limited by its inability to assess regional function and has lower sensitivity in detecting early cardiac impairments (1-3).

Strain is a parameter that can be used to assess myocardial regional deformation. By measuring strain in the longitudinal, circumferential, and radial orientations, we can study cardiac function both regionally and globally (4). Myocardial strain imaging thus serves as a valuable diagnostic and prognostic tool for evaluating cardiac abnormalities (5).
Currently, cardiovascular magnetic resonance imaging (CMR) serves as the gold standard for the quantification of biventricular strain (6). Over the years, there have been a number of different CMR methods used for assessing strain, including myocardial tagging (MT), displacement encoding with stimulated echoes (DENSE), and most recently, feature tracking (FT) (4). Specifically, CMR FT has emerged as a simple post-processing method for quantifying strain (7). It is becoming the preferred technique in many research and clinical settings for its relative ease in analyzing a large amount of cine data. However, despite its relative popularity, CMR FT has disadvantages including inter-vendor differences, potentially low spatial 

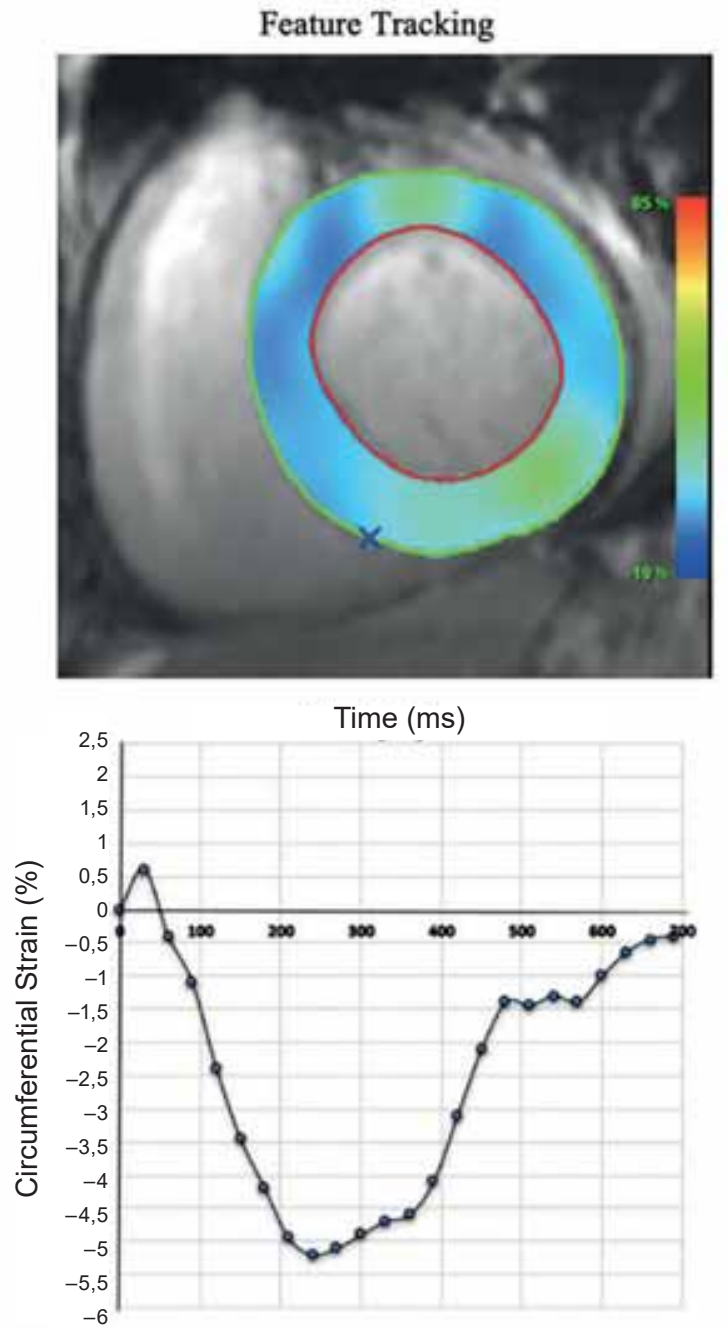
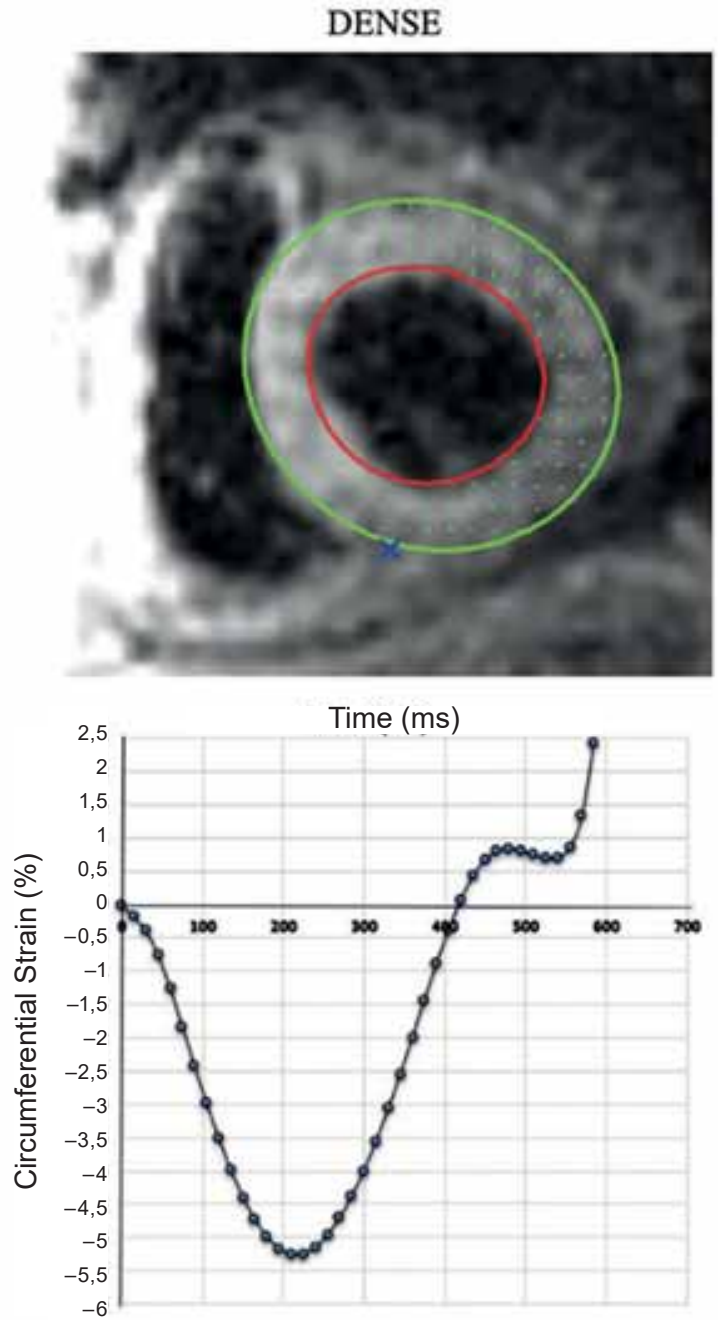

FIGURE 1. Strain Analysis by Feature Tracking and DENSE. Strain analysis on the same patient. The top panels show strain analysis by feature tracking and DENSE using SuiteHEART (Neosoft, Pewaukee, WI, USA). In feature-tracking, the endocardial and epicardial borders are marked by red and green contours, respectively, with myocardium color coded with strain values. In DENSE, the endocardial and epicardial borders are marked by red and green contours, respectively, with myocardium depicting the 2D displacement field. The bottom panel shows the respective circumferential strain curves derived from feature tracking and DENSE. DENSE: Displacement encoding with stimulated echoes.

and temporal resolution, and suboptimal correlation with other CMR strain techniques.

The aim of this review is to summarize the general principles of CMR FT and describe the advantages and disadvantages of its widespread use. Furthermore, we aim to provide a recommendation for future directions for improving the current landscape of CMR FT.

\section{General Principles of Feature Tracking}

CMR FT is based on a pattern-matching technique of tracking unique "features" across different images in a cardiac cycle (8). First, the endocardial and epicardial borders are traced at end diastole, the slice representing the end of diastolic filling and the best separation between the blood pool and myocardial tissue. The CMR FT software algorithm then automatically identifies pixels using a combination of information including tissue-cavity interface, spatial coherence, and signal-to-noise ratios (9). A pixel is identified in one frame and is followed in the next successive frames, leading to tracking of myocardial deformations (10).

In order to accurately identify and track the displacement of border, tracking is performed orthogonal to the cavity-tissue boundary. Transmural cuts are drawn orthogonal to the cavity-tissue border and columns of pixels are chosen along the transmural line throughout the cardiac cycle. In each successive frame, the displacement of the pixels in the columns are measured and can be displayed in a 2D representation of change in displacement versus time (11). 


\section{The Good - Acquisition}

CMR FT quantifies regional deformations in the myocardium by post-processing cine images, which are routinely acquired in CMR exams (12). Its main advantage is the lack of need for additional image acquisition. CMR myocardial tagging (MT) is the gold standard technique for the measurement of myocardial strain and other strain techniques including 2D speckle-tracking echocardiography (STE) have been validated against MT (6). Radiofrequency saturation pulses are introduced in two orthogonal planes to the myocardium, effectively creating a grid of myocardial tags on the myocardium that can be tracked during the cardiac cycle. The tracking of myocardial deformations allows for the quantification of strain. Various other strain acquisition techniques are available including DENSE and strain encoding magnetic resonance imaging (SENC) (9). While these techniques might be the most accurate non-invasive means of measuring myocardial strain, they require specific acquisition and post-processing, rendering them predominantly as a research tool.

CMR FT does not require additional image acquisition for strain analysis as it utilizes the standard steady-state free procession (SSFP) cine images for analysis (8). Since SSFP is the routine imaging sequence that has been the corner stone of CMR imaging for more than 20 years, there is a vast availability of data in a variety of diseases for analysis (8). In spite of the differences between the strain values produced by FT software packages and by gold standard techniques such as tagging and DENSE (Figure 1), FT usage will continue to expand in research and clinical settings.

\section{The Bad - Reproducibility and Resolution}

CMR MT, DENSE, and FT all quantify strain differently and the few studies done to ascertain FT reproducibility and agreement with MT have shown that significant differences between the stain values obtained with the different methods (13-15). Acquisition-based techniques (MT, DENSE) were also shown to have better reproducibility and agreement in segmental strain values when compared with FT (15). Although global strain measurements have better reproducibility than regional or segmental strain, some FT software packages have been shown to consistently and systematically overestimate strain values (16). In globally measured strains, longitudinal and circumferential strains have also been shown to have superior reproducibility and agreement than radial strain (17). Since intra- and inter-observer reproducibility of $\mathrm{FT}$ is not as good as MT, the values produced by FT may not be as sensitive as MT or DENSE in early disease detection.

Any factors that affect cine imaging would also affect FT. CMR FT is limited in spatial and temporal resolu- tion as compared to MT. While MT uses the inherent magnetization properties of the myocardium, FT must rely on the already acquired pixel size in tracking deformations (18). Displacements that are smaller than the designated pixel size will not be detectable by the FT software and thus affect post-processed strain values. Pixel sizes that are too big (spatial resolution that are too low) may not capture regional abnormalities or may underestimate the true extent of myocardial shortening. Furthermore, depending on the spatial resolution, blood pool near the tissue-cavity border may interfere with the tracking software and produce inaccurate strain values with associated deformations. Temporal resolutions that are too low may not capture peak strain value properly and thus underestimate strain. Minimal spatial and temporal resolution has not been standardized in SSFP acquisition for FT analysis. In STE, a minimal of 30 frames per second is required to post-process for strain (19). This is equivalent of $33 \mathrm{~ms}$ temporal resolution for CMR. In addition, studies with SSFP images acquired at different spatial or temporal resolutions cannot be simply combined for analysis due to differences in strain values that can be obtained from the SSFP images just based on different spatial or temporal resolutions.

Whether or not the cine imaging was obtained pre or post contrast injection can also affect FT values (20). FT algorithms depend on the clear contrast between blood and myocardium in SSFP images. Due to decreased contrast-to-noise ratios from extracellular contrast agents, FT on cine images obtained post-contrast have lower strain values and are also significantly less reproducible than pre-contrast strain values (20).

\section{The Ugly - Inter-vendor Differences in Post-Processing and Lack of Standardization}

Because of the relative ease of FT post-processing, there are currently more than 10 available CMR FT software packages in research and clinical settings. Different software packages such as TomTec (TomTec Imaging Systems, Germany) and CVI42 (Circle Cardiovascular Imaging, Calgary, Canada), use different algorithms in their post-processing package to quantify strain values. Due to the proprietary nature of the commercial algorithms, the exact methodology of how they obtain stain values is unclear, making it difficult to directly compare and understand the differences in values produced by different software packages. A recent study comparing inter-vendor agreements and reproducibility between TomTec and CVI42 found that there were significant inter-vendor variability (20).

The inter-vendor differences in CMR FT software packages will affect the strain measurements obtained. Although CMR is recognized as the gold standard for 
the quantification of strain, one study reported that STE provided stronger prognostic value in predicting cardiovascular mortality in heart failure with preserved ejection fraction patients (21). The discrepancy between the prognostic value of STE and CMR may be due to the selection of certain post-processing FT software and not the imaging modality. The fact that there are so many different FT software has further amplified this problem. This underscores the need for standardization of SSFP acquisition parameters, post-processing algorithms, and software specific reference standards for strain values in FT software packages.

\section{Conclusion}

Myocardial strain measurements provide additive diagnostic and prognostic information to conventional cardiac functional markers such as EF and ventricular volumes. CMR FT provides an easy way to obtain strain values through SSFP cine images without any additional specific image acquisition. CMR FT post-processing is also fast, gaining it widespread popularity in research and clinical settings. However, FT is hindered by a number of disadvantages as well. FT has lower reproducibility and resolution than conventional gold standard MT or DENSE techniques. More importantly, FT is vendor-dependent. There is significant variability of strain measurements between different software packages, which may affect the generalizability of clinical outcomes of data analyzed by FT. Future standardization of FT software practices must be implemented in order to continue widespread adoption of FT in clinical and research settings.

\section{References}

1. Almutairi HM, Boubertakh R, Miquel ME, Petersen SE. Myocardial deformation assessment using cardiovascular magnetic resonance-feature tracking technique. Br J Radiol 2017; 90(1080): 20170072. doi: 10.1259/bjr. 201700722.

2. Scatteia A, Baritussio A, Bucciarelli-Ducci C. Strain imaging using cardiac magnetic resonance. Heart Fail Rev 2017; 22(4): 465-476. doi: 10.1007/s10741-017-9621-8

3. Amzulescu MS, De Craene M, Langet $\mathrm{H}$, et al. Myocardial strain imaging: review of general principles, validation, and sources of discrepancies. Eur Heart J Cardiovasc Imaging 2019; 20(6): 605619. doi: $10.1093 /$ ehjci/jez041

4. Kurt M, Tanboga IH, Aksakal E. Two-Dimensional Strain Imaging: Basic principles and Technical Consideration. EAJM 2014; 46(2): 126-130. doi: 10.5152/eajm.2014.28

5. Voigt J-U, Cvijic M. 2- and 3-Dimensional Myocardial Strain in Cardiac Health and Disease. JACC Cardiovasc Imaging 2019; 12(9): 1849-1863. doi: 10.1016/j.jcmg.2019.01.044

6. Kanagala P, Cheng ASH, Singh A, et al. Diagnostic and prognostic utility of cardiovascular magnetic resonance imaging in heart failure with preserved ejection fraction - implications for clinical trials. J Cardiovasc Magn Reson 2018; 20(1): 4. doi: 10.1186/s12968017-0424-9
7. Rahman ZU, Sethi P, Murtaza G, et al. Feature tracking cardiac magnetic resonance imaging: A review of a novel non-invasive cardiac imaging technique. World J Cardiol 2017; 9(4): 312-319. doi: 10.4330/wjc.v9.i4.312

8. Salerno M. Feature Tracking by CMR: A "Double Feature"? JACC Cardiovasc Imaging 2018; 11(2 Pt 1): 206-208. doi: 10.1016/j. jcmg.2017.01.024

9. Schuster A, Hor KN, Kowallick JT, Beerbaum P, Kutty S. Cardiovascular Magnetic Resonance Myocardial Feature Tracking: Concepts and Clinical Applications. Circ Cardiovasc Imaging 2016; 9(4). doi: 10.1161/CIRCIMAGING.115.004077

10. Ruijsink B, Puyol-Antón E, Oksuz I, et al. Fully Automated, Quality-Controlled Cardiac Analysis From CMR: Validation and Large-Scale Application to Characterize Cardiac Function. JACC Cardiovasc Imaging July 2019. doi: 10.1016/j.jcmg.2019.05.030

11. Hor KN, Baumann R, Pedrizzetti G, et al. Magnetic Resonance Derived Myocardial Strain Assessment Using Feature Tracking. JoVE 2011; (48): 2356. doi: 10.3791/2356

12. Kowallick JT, Kutty S, Edelmann F, et al. Quantification of left atrial strain and strain rate using cardiovascular magnetic resonance myocardial feature tracking. J Cardiovasc Magn Reson 2015; 17(S1): P66, 1532-429X-17-S1-P66. doi: 10.1186/1532-429X-17-S1-P66 13. Cao JJ, Ngai N, Duncanson L, Cheng J, Gliganic K, Chen Q. A comparison of both DENSE and feature tracking techniques with tagging for the cardiovascular magnetic resonance assessment of myocardial strain. J Cardiovasc Magn Reson 2018; 20(1): 26. doi: 10.1186/s12968-018-0448-9

14. Vo HQ, Marwick TH, Negishi K. MRI-Derived Myocardial Strain Measures in Normal Subjects. JACC Cardiovasc Imaging 2018; 11 (2 Pt 1): 196-205. doi: 10.1016/j.jcmg.2016.12.025

15. Bucius P, Erley J, Tanacli R, et al. Comparison of feature tracking, fast-SENC, and myocardial tagging for global and segmental left ventricular strain. ESC Heart Failure December 2019: ehf2.12576. doi: 10.1002/ehf2.12576

16. Augustine D, Lewandowski AJ, Lazdam M, et al. Global and regional left ventricular myocardial deformation measures by magnetic resonance feature tracking in healthy volunteers: comparison with tagging and relevance of gender. J Cardiovasc Magn Reson 2013; 15: 8. doi: 10.1186/1532-429X-15-8

17. Doerner J, Bunck AC, Michels G, Maintz D, Baeßler B. Incremental value of cardiovascular magnetic resonance feature tracking derived atrial and ventricular strain parameters in a comprehensive approach for the diagnosis of acute myocarditis. European Journal of Radiology 2018; 104: 120-128. doi: 10.1016/j.ejrad.2018.05.012 18. Heinke R, Pathan F, Le M, et al. Towards standardized postprocessing of global longitudinal strain by feature tracking - OptiStrain CMR-FT study. BMC Cardiovasc Disord 2019; 19(1): 267. doi: 10.1186/s12872-019-1255-4

19. Rösner A, Barbosa D, Aarsæther E, Kjønås D, Schirmer $H$, D'hooge J. The influence of frame rate on two-dimensional speckle-tracking strain measurements: a study on silico-simulated models and images recorded in patients. Eur Heart J Cardiovasc Imaging 2015; 16(10): 1137-1147. doi: 10.1093/ehjci/jev058

20. Kuetting DLR, Dabir D, Homsi R, et al. The effects of extracellular contrast agent (Gadobutrol) on the precision and reproducibility of cardiovascular magnetic resonance feature tracking. J Cardiovasc Magn Reson 2016; 18(1): 30. doi: 10.1186/s12968-016-0249-y 21. Houard L, Benaets M-B, de Meester de Ravenstein C, et al. Additional Prognostic Value of 2D Right Ventricular Speckle-Tracking Strain for Prediction of Survival in Heart Failure and Reduced Ejection Fraction: A Comparative Study With Cardiac Magnetic Resonance. JACC Cardiovasc Imaging February 2019. doi: 10.1016/j. jcmg.2018.11.028 